\title{
ESTRUTURA DINÂMICA DAS CONTRACORRENTES EQUATORIAIS DO OCEANO ATLÂNTICO AO LONGO DE 44W
}

\author{
Domingos Fernandes Urbano Neto, ${ }^{1}$ Ilson Carlos Almeida da Silveira ${ }^{2}$ \\ Submetido em 15, julho, 2003/Aceito em 13, abril, 2004 \\ Submited July 15, 2003/Accepted April 13, 2004
}

\section{RESUMO}

Estudos teóricos ou baseados em modelagem numérica indicam que a estrutura vertical do oceano tropical é dominada pelos dois primeiros modos baroclínicos. Entretanto, faltam resultados quantitativos que mostrem efetivamente a dominância destes modos através de comparações com observações de velocidade no Oceano Atlântico. Neste artigo, utilizamos perfilagens de Conductivity-Temperature-Depth (CTD) simultâneas às de velocidade (Pegasus), realizadas durante 0 Projeto Western Tropical Atlantic Experiment (WESTRAX), para avaliar a estrutura modal das contra-correntes equatoriais ao longo de 44W: as Contracorrentes Norte Equatorial (CCNE) e Subcorrente Norte Equatorial (SNE). Os resultados mostram que, de fato, os dois primeiros modos barodínicos são mais importantes que o modo barotrópico e os demais modos baroclínicos. A soma dos três primeiros modos dinâmicos explica cerca de $50 \%$ da estrutura vertical das correntes. Talvez devido à proximidade da camada de limite oeste oceânica, e portanto sítio de origem tanto da CCNE quanto da SNE, os resultados apresentam nítida variação sazonal. De fato, os três primeiros modos baroclínicos respondem por cerca de $63 \%$ da estrutura vertical de velocidade observada, sendo que o primeiro modo é responsável por 35-45\% do sinal medido. Esses resultados corroboram aqueles encontrados para 0 cruzeiro de outono boreal em estudos anteriores. No entanto, para os cruzeiros de inverno boreal, onde uma estrutura de velocidade apresenta-se mais complexa, é necessária a introdução de maior número de modos dinâmicos. Tanto para 0 cruzeiro de outono quanto para 0 de inverno houve necessidade seis modos dinâmicos para reproduzir $63 \%$ da estrutura de velocidade observada. Os três primeiros modos dinâmicos são responsáveis por 30-40\% do sinal observado. Estudos comparativos entre a estrutura dinâmica das correntes equatoriais em 44W e em porções mais centrais da bacia do 0ceano Atlântico, devem ser conduzidos para estabelecer o papel dos modos de mais alta ordem e sua relação com a camada limite oeste.

Palavras-chave. Dinâmica Equatorial; Modos Dinâmicos; Meso-escala; Correntes Equatoriais.

\section{ABSTRACT}

It is commonly mentioned in the literature that the first and second baroclinic modes dominate the vertical structure in the tropical ocean. However, most of these studies refer to either theoretical or numerical modeling. There are no quantitative results that confirm the two internal mode dominance through comparisons with velocity observations in the Tropical Atlantic. In this article, we use hydrographic (CTD) and velocity (PEGASUS) profiles obtained during the "Western Tropical Atlantic Experiment-WESTRAX" to evaluate the modal structure of the equatorial currents along 44W, in particular, the North Equatorial Countercurrent (NECC) and the North Equatorial Undercurrent (NEUC). The results show that indeed the first two baroclinic modes explain about $50 \%$ of the vertical structure of the currents on average. We also find indication of a clear seasonal variation on the modal composition, a probable consequence of the proximity of the study region to the western boundary layer, and therefore, the source of both NECC and NEUC. As a mather of fact, the first three dynamical mode account for about $63 \%$ of the observed velocity vertical structure, while the first mode alone is responsible for $35-45 \%$ of the measured velocity. These results reasonably agree with previous studies also using the fall WESTRAX cruise. However, the more complex boreal winter cruise velocity patterns yield an increased number of dynamical modes to reconstitute the velocity field. For the both fall and winter cruises, we need six dynamical modes to reproduce $63 \%$ of the observed velocity structure. The first three dynamical mode are responsible for 30-40\% of the observed signal. A comparative study of the dynamical structure of the zonal equatorial currents at $44 \mathrm{~W}$ and at more central portions of the Atlantic 0 cean basin should be conducted in order to establish the role of the higher order modes and its relation with the western boundary layer.

Keywords: Equatorial Dynamics; Dynamical Modes; Mesoescale; Equatorial Currents.

\footnotetext{
Laboratório de Dinâmica Oceânica - Departamento de Oceanografia Física, Química e Geológica - Instituto Oceanográfico - Universidade de São Paulo - Praça do Oceanográfico, 191 Cidade Universitária - CEP: 05508-900 - São Paulo - SP, Brasil, ou - Caixa Postal 9075 - 01065-970 - São Paulo - SP, Brasil - Tel.: (11) 3091-6552 - FAX: (11) 3091 -6610. E-mail: dfurbano@io.usp.br.

2 Laboratório de Dinâmica Oceânica - Departamento de Oceanografia Física, Química e Geológica - Instituto Oceanográfico - Universidade de São Paulo - Praça do Oceanográfico, 191Cidade Universitária - CEP: 05508-900 - São Paulo - SP, Brasil, ou - Caixa Postal 9075 - 01065-970 - São Paulo - SP, Brasil - Tel.: (11) $3091-6552$ - FAX: (11) 3091 -6610. E-mail: ilson@io.usp.br
} 


\section{INTRODUÇÃOO}

A região equatorial oeste do Oceano Atlântico tem sido fortemente investigada ao longo das últimas décadas. Correntes de Contorno Oeste desempenham um importante papel como elo de ligação na circulação termo-halina global. Processos como o de retroflexão do sistema Corrente Norte do Brasil (CNB), além de alimentarem em diferentes níveis picnoclínicos a Contracorrente Norte Equatorial (CCNE) e a Subcorrente Norte Equatorial (SNE), emitem anéis de núcleo quente em direção ao Mar do Caribe. Parte da Corrente Norte Equatorial (CNE), fluindo em direção à borda do continente sul-americano, reverte ciclonicamente e contribui de forma significante no transporte da CCNE. Torna-se evidente a complexidade na estrutura vertical das correntes desta região equatorial. Como conseqüência desta estrutura vertical, Philander (1989) resume que as regiões equatoriais, não só do Oceano Atlântico, se contrapõem às regiões subtropicais, apresentando os dois primeiros modos baroclínicos como dominantes, e o modo barotrópico possuindo menor importância. No entanto, estudos realizados a respeito da estrutura modal são baseados em resultados obtidos através de modelagem numérica aplicada a oceanos idealizados, baseados em modelos teóricos, ou em interpretação de simulações numéricas de modelagem processual.

Lighthill (1969) usou modelagem numérica aplicada a região tropical, fazendo uso de uma estratificação básica mais próxima de uma situação real, onde a termoclina se apresenta menos acentuada, mostrou que o primeiro modo barodinico é 0 mais importante na estrutura modal de correntes equatoriais. Estudos analíiticos realizados por Cane e Sarachik (1976); Cane e Sarachik (1977); Cane e Sarachik (1979) utilizam um modelo de águas rasas de primeiro modo baroclínico para examinar a geração de correntes lineares em um oceano equatorial. Cane (1979) estudou a resposta de um oceano equatorial sob influência do vento usando um modelo numérico de 1-1/2 camada. Esta estrutura de massa, obviamente implica num primeiro modo barodínico importante.

No modelo de equações primitivas de Philander e Pacanowski (1980), uma estratificação básica é assumida e representada por um perfil de frequiência de Brunt-Väisälä idealizado. Segundo os autores, os modos naturais de oscilação desempenham um papel central no ajustamento do oceano às variações dos ventos de superfície. Afirmam ainda, que o segundo modo baroclínico é dominante neste processo por apresentar 0 maior valor de amplitude modal em regiões próximas à superfície, e que devido a isso, qual quer destúrbio nas camadas superiores do oceano resulta em uma grande projeção dentro do segundo modo baroclínico, que por esta razão, pode ser usado para representar a estrutura dinâmica do oceano tropical, não apenas no centro da bacia oceânica de baixas latitudes, mas em qualquer parte desta.
Mais recentemente, McClean e Klinck (1995), usando o modelo CME (WOCE Community Modeling Effort general circulation), mostraram que a região da CCNE é dominada pelo primeiro e segundo modos baroclínicos. Patti (2001) usou um modelo de três camadas - e portanto, de três modos dinâmicos - para reproduzir o processo de emissão de vórtices pela CNB, através da colisão de ondas de Rossby de primeiro e segundo modos na região do contorno oeste.

Em termos de análise da estrutura de velocidade observada no Atlântico Tropical, Bourlès e outros (1999) descreveram observações de perfilagem de velocidade ao longo de $44 \mathrm{~W}$ a partir dos cruzeiros WESTRAX e um cruzeiro hidrográfico realizado pela NOAA (National Oceanic and Atmospheric Administration), em agosto de 1989 (WILSON et al., 1994). Estes autores examinaram 0 campo de velocidade na camada superior do oceano e apresentaram características inéditas da CCNE. 0 resultado mais importante deste estudo foi a descrição de um comportamento quase-sazonal do sistema zonal de correntes equatoriais. Durante os cruzeiros de outono/verão boreais, as CCNE e SNE estão fusionadas e apresentam um núcleo em subsuperfície. Durante os cruzeiros de inverno boreal, estas correntes estão separadas por pelo menos três graus de latitude. Estes resultados indicam a existência de uma estrutura modal baroclínica complexa, provavelmente devido à proximidade do contorno oeste.

Silveira e outros (2000), realizando uma comparação entre os modos de variabilidade vertical estatísticos (Depth Empirical Orthogonal Functions - DEOFs) e os modos dinâmicos, calculados a partir de observaç̃oes WESTRAX de outono boreal de 1990, da região de retroflexão da CNB, mostram que os dois primeiros modos estatísticos dessa função explicam 90\% da variância na estrutura vertical. Um ajuste ótimo para os modos dinâmicos nos modos DEOF sugere que o primeiro e segundo modos dessa função são dominados (dinamicamente) pelos primeiro e segundo modos baroclínicos, respectivamente. Silveira et al (2000) mostraram ainda que a soma dos primeiros três modos dinâmicos é responsável por 75\% da estrutura vertical dos dois primeiros modos da DEOF. Dessa forma, formularam um modelo de três camadas para representar o sistema de retroflexão da CNB.

Os resultados evidenciados anteriormente conduzem às seguintes questôes:

- São os primeiro e segundo modos baroclínicos, como dito por Philander (1989), mais relevantes que o modo barotrópico na região equatorial do Oceano Atlântico Tropical Oeste?

- É a afirmação de Philander e Pacanowski (1980) - de que o segundo modo baroclínico é dominante - realmente válida? Pode este modo ser usado como representante da estrutura dinâmica para qualquer porção do Oceano Atlântico Tropical? 
- Os três primeiros modos dinâmicos (barotrópico e dois primeiros baroclínicos) foram suficientes para reproduzir de forma satisfatória 0 processo de retroflexão do sistema CNB (SILVEIRA et al., 2000). 0 mesmo pode ser aplicado para o sistema de correntes zonais ao longo de $44^{\circ} \mathrm{W}$ ?

- De que forma o núcleo de subsuperfície da CCNE, relatado por Bourlès e outros (1999), está relacionado à estrutura modal da região?

Buscando responder essas questões, uma análise de modos normais foi realizada para um dos únicos conjuntos de dados quasesinóticos que envolvem medições diretas de correntes, existente na região tropical oeste do Oceano Atlântico, o conjunto WESTRAX. Observaç̧es hidrográficas e de velocidade obtidas de forma simultânea, tornam possível uma investigaç̧ão detalhada e precisa a respeito da estrutura dinâmica ao longo da seção de 44 W, ou seja, uma porção do oceano equatorial localizada entre dois importantes processos dinâmicos: a retroflexão do sistema CNB e o sistema de correntes zonais equatoriais.

\section{CONJUNTO DE OBSERVAÇÕES}

0 conjunto de dados consiste de componentes de velocidade obtidas por perfilador de correntes PEGASUS (SPAIN et al., 1981) e amostragens simultâneas, em toda coluna de água, de temperatura e salinidade medidas através de (TD (Conductivity-Temperature-Depth). Estes dados foram obtidos durante o Western Tropica/Atlantic Experiment - WESTRAX (BROWN et al., 1992). 0 programa experimental completo consiste de cinco cruzeiros realizados de 1990 a 1991 (Tabela 1). Neste trabalho, limitamo-nos à análise dos quatro primeiros cruzeiros.

Tabela 1 - Sumário das observações WESTRAX.

Table 1 - Summary of the WESTRAX observations.

\begin{tabular}{cccc}
\hline Cruzeiro & Período & Ano & Estação Boreal \\
\hline Jan90 & 31 Jan - 19 Fev & 1990 & Inverno \\
Set90 & 17 Set - 10 Out & 1990 & Outono \\
Jan91 & 16 Jan - 30 Jan & 1991 & Inverno \\
Jun91 & 20 Jun - 03 Jul & 1991 & Verão \\
Set91* & 07 Set - 17 Set & 1991 & Outono \\
\hline
\end{tabular}

*Dados deste cruzeiro não foram utilizados neste estudo.

0 Projeto WESTRAX amostrou a região do Oceano Atlântico compreendida pela região à oeste de $44^{\circ} \mathrm{W}$ e ao norte da costa sulamericana, até a latitude de 15N (Figura 1). As perfilagens foram dispostas em um conjunto de radiais, formando uma malha hidrográfica. Para este trabalho, foram analisadas as estações ao longo de $44^{\circ} \mathrm{W}$, uma vez que registraram 0 sinal do sistema zonal de correntes equatoriais próximo à sua região de origem, ou seja, a região entre a borda oeste e 0 centro da bacia.

Entre os mais recentes trabalhos utilizando o conjunto de dados provenientes do Projeto WESTRAX, encontra-se o de Bourlès e outros (1999). Os autores examinaram 0 campo de velocidade da camada superior do oceano, utilizando dados de ADCP de bordo (Shipboard Acoustic Doppler Current Profiler) e de CTD02 (Conductivity-TemperatureDepth-Oxygen). 0 oceano superior foi dividido em porções, onde as fronteiras verticais foram, além da interface oceano-atmosfera, as superfícies das isopicnais de 24,50 e 26,75 kg m. Seções verticais de velocidade ao longo de $44 \mathrm{~W}$ foram apresentadas e descritas em detalhe.
Aqui, apresentamos seções da componente zonal de velocidade análogas àquelas apresentadas por Bourlès e outros (1999), porém utilizando os dados do perfilador PEGASUS (Figura 2). Ambas isopicnais foram sobrepostas aos contornos de velocidade e são indicadas pela linha espessa. As isotacas apresentam intervalo de $0.1 \mathrm{~m} \mathrm{~s}$, com valores positivos indicando fluxo para leste e negativos para oeste. A localização das estações é sinalizada pelos círculos desenhados na parte superior do campo.

0 painel da Figura 2 mostra claramente duas fases distintas no comportamento das correntes CCNE e SNE. Durante o período de inverno boreal (cruzeiros de jan90 e jan91; Tabela 1), o núcleo da CCNE está centrado em 3,2N. A SNE encontra-se deslocada para norte, com seu núcleo posicionado além da fronteira norte do campo, em 1990, e centrado em 6,8N, em 1991. Estão notoriamente separados por uma distância mínima de quatro graus de latitude. Por outro lado, nos cruzeiros de primavera e outono (set90 e Jun91), as duas correntes estão alinhadas verticalmente com um forte núcleo posicionado acima da isopicnal de 


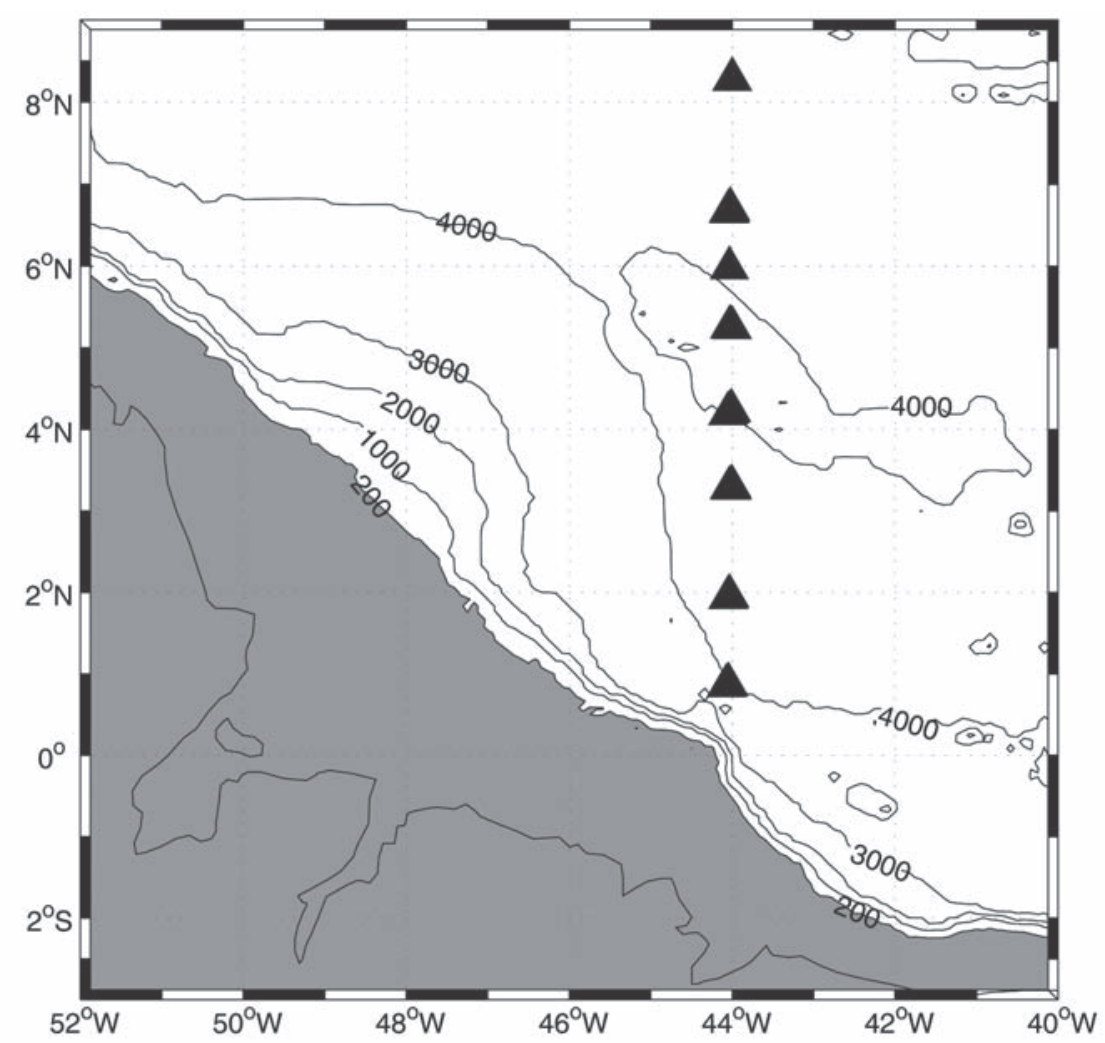

Figura 1 - Região de estudo. As posições das estações hidrográficas realizadas por PEGASUS e CTD estão representadas pelos triângulos.

Figure 1 - The study area. The location of the hydrographic/PEGASUS and CTD stations are represented by dark triangles.

24,5 kg m (Figura 2 linha espessa). Bourlès e outros (1999) descrevem a presença de uma nítida separação vertical entre os núcleos dessas correntes durante 0 cruzeiro Jun91. Uma descontinuidade na isopicnal de 26,75 (Figura 2, linha espessa), em 3,2N, indica a presença da SNE durante esses cruzeiros.

Ao norte do sistema CCNE existe um escoamento para oeste associado à borda sul da CNE. Esta corrente aparece mais evidente durante os cruzeiros de primavera e outono (de set90 e jun91), quando a CCNE desloca-se para sul devido ao posicionamento da Zona de Confluência Intertropical (ZCIT).

Como as perfilagens de PEGASUS cobriram toda coluna de água, ao contrário dos dados de ADCP que atingem profundidades de no máximo 500 metros, apresentamos painéis complementares que mostram a circulação abaixo dos 1000 metros de profundidade. Atentamos que estes gráficos apresentam escala vertical distinta da porção superior, no entanto, fica evidente um padrão de velocidades baixas na maior parte da coluna de água e que as regióes de maior cisalhamento encontramse confinadas aos primeiros 1000 metros de profundidade.

\section{DECOMPOSIÇÃO MODAL}

0 cenário aqui envolvido consiste de um oceano sob aproximação hidrostática, continuamente estratificado, com tampa rígida e fundo plano como condições de contorno para a superfície e fundo, respectivamente. A estrutura vertical de velocidade foi decomposta em modos dinâmicos, seguindo formalismo apresentado por Silveira e outros (2000). As componentes da equação do movimento linearizadas, sob aproximação de Boussinesq, podem ser desacopladas em estruturas vertical e horizontal, aplicando-se o método de separação de variáveis, descrito em LeBlond e Mysak (1978):

$$
\begin{aligned}
& \mathrm{p}(\mathrm{x}, \mathrm{y}, \mathrm{z}, \mathrm{t})=\mathrm{P}_{\mathrm{i}}(\mathrm{x}, \mathrm{y}, \mathrm{t}) \mathrm{F}_{\mathrm{i}}(\mathrm{z}), \\
& \mathrm{u}(\mathrm{x}, \mathrm{y}, \mathrm{z}, \mathrm{t})=\mathrm{U}_{\mathrm{i}}(\mathrm{x}, \mathrm{y}, \mathrm{t}) \mathrm{F}_{\mathrm{i}}(\mathrm{z}), \\
& \mathrm{w}(\mathrm{x}, \mathrm{y}, \mathrm{z}, \mathrm{t})=\mathrm{W}_{\mathrm{i}}(\mathrm{x}, \mathrm{y}, \mathrm{t}) \frac{1}{\mathrm{~N}^{2}(\mathrm{z})} \frac{\mathrm{dF}_{\mathrm{i}}(\mathrm{z})}{\mathrm{dz}} .
\end{aligned}
$$



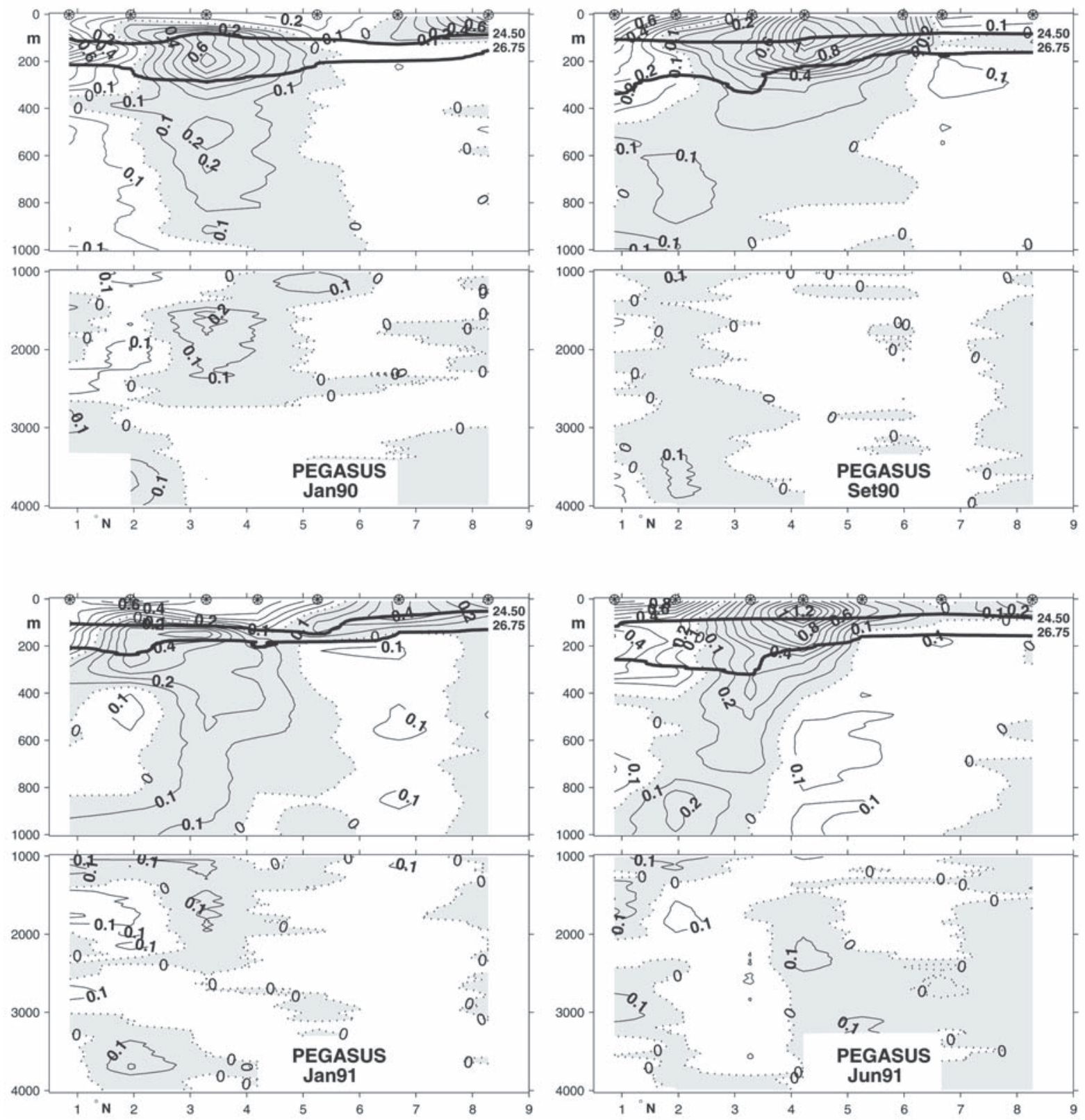

Figura 2 - Componente zonal de velocidade ao longo de $44^{\circ} \mathrm{W}$ obtido por PEGASUS. Os contornos possuem intervalo de $0.1 \mathrm{~m} \mathrm{~s} \mathrm{~s}^{-1}$. As profundidades estão expressas em metros e as linhas espessas indicam as isopicnais de $24,50 \mathrm{e} 26,75 \mathrm{~kg} \mathrm{~m}^{-3}$.

Figure 2 - Zonal velocities at 44 $\mathrm{W}$ measured by PEGASUS. The contour intervals correspond to $0.1 \mathrm{~m} \mathrm{~s}^{\prime}$. The depth is in meters and the thick lines are the isopicnals of 24.50 and $26.75 \mathrm{~kg} \mathrm{~m}^{3}$. 
onde $\mathrm{P}_{\mathrm{i}^{\prime}} \mathrm{V}_{\mathrm{i}}$ e $\mathrm{W}_{\mathrm{i}}$ são as amplitudes do i-ésimo modo vertical da pressão e das velocidades zonal e vertical, respectivamente; $\mathrm{F}_{\mathrm{i}}$ é 0 iésimo modo vertical; e N é a freqüência de estratificação ou Freqüência de Brünt-Väisälä.

Os modos verticais satisfazem a equação de autovalores $(\lambda \mathrm{i})$

$$
\frac{\mathrm{d}}{\mathrm{dz}} \frac{1}{\mathrm{~N}^{2}} \frac{\mathrm{dF}_{\mathrm{i}}}{\mathrm{dz}}+\lambda_{\mathrm{i}} \mathrm{F}_{\mathrm{i}}=0
$$

As condições de contorno para $\mathrm{F}_{1}$ são fundo plano e tampa rígida em superfície:

$$
\frac{\mathrm{dF}_{\mathrm{i}}}{\mathrm{dz}}=0, \quad \mathrm{z}=-\mathrm{H} \quad \mathrm{z}=0 \text {, }
$$

A Equação (4) juntamente com as condições de contorno representadas por (5) constituem um problema de autovalores para $\lambda_{i}$. Assim, existe um número infinito de soluções $\mathrm{F}_{\mathrm{i}}(\mathrm{z})$, para $\mathrm{i}=0,1,2, \ldots$, cada uma associada a um autovalor $\lambda_{i}$ real e discreto. Portanto, os modos dinâmicos são funcionais da freqüência de Brünt-Väisälä.

As amplitudes $\mathrm{U}_{\mathrm{i}}$ são obtidas através da projeção dos perfis de velocidades observadas por PEGASUS nos modos dinâmicos, através da integração:

$$
\mathrm{U}_{\mathrm{i}}=\frac{1}{\mathrm{H}} \int_{0}^{\mathrm{H}}(\mathrm{u}) \mathrm{F}_{\mathrm{i}} \mathrm{dz}
$$

onde u é a componente zonal da velocidade observada.

Nas seções a seguir descreve-se uma discussão sobre a estrutura de estratificação da região WESTRAX e cálculo dos modos dinâmicos associados a esta.

\section{ESTRUTURA VERTICAL DA ESTRATIFICAÇÃO}

Perfis médios sazonais de densidade potencial $\left(\sigma_{\theta}\right)$ foram calculados a partir dos dados de salinidade e temperatura obtidos por CTD. Um perfil médio dos quatro cruzeiros também foi calculado e denominado de média anual. Perfis de Freqüência de Brunt-Väisälä (N) foram obtidos através da equação:

$$
\mathrm{N}^{2}(\mathrm{z})=\frac{-\mathrm{g}}{\rho_{0}} \frac{\partial \sigma_{\theta}}{\partial \mathrm{z}},
$$

onde $g$ é a aceleração devido à gravidade, $\rho_{0}$ é a densidade potencial média da água do mar. Os perfis de $\mathrm{N}$ e da densidade convencional $\sigma_{\theta}$ estão dispostos na Figura 3. Os perfis sazonais das freqüências de empuxo estão representados (Figura 4), pela curva mais espessa. A curva mais delgada representa o perfil médio.

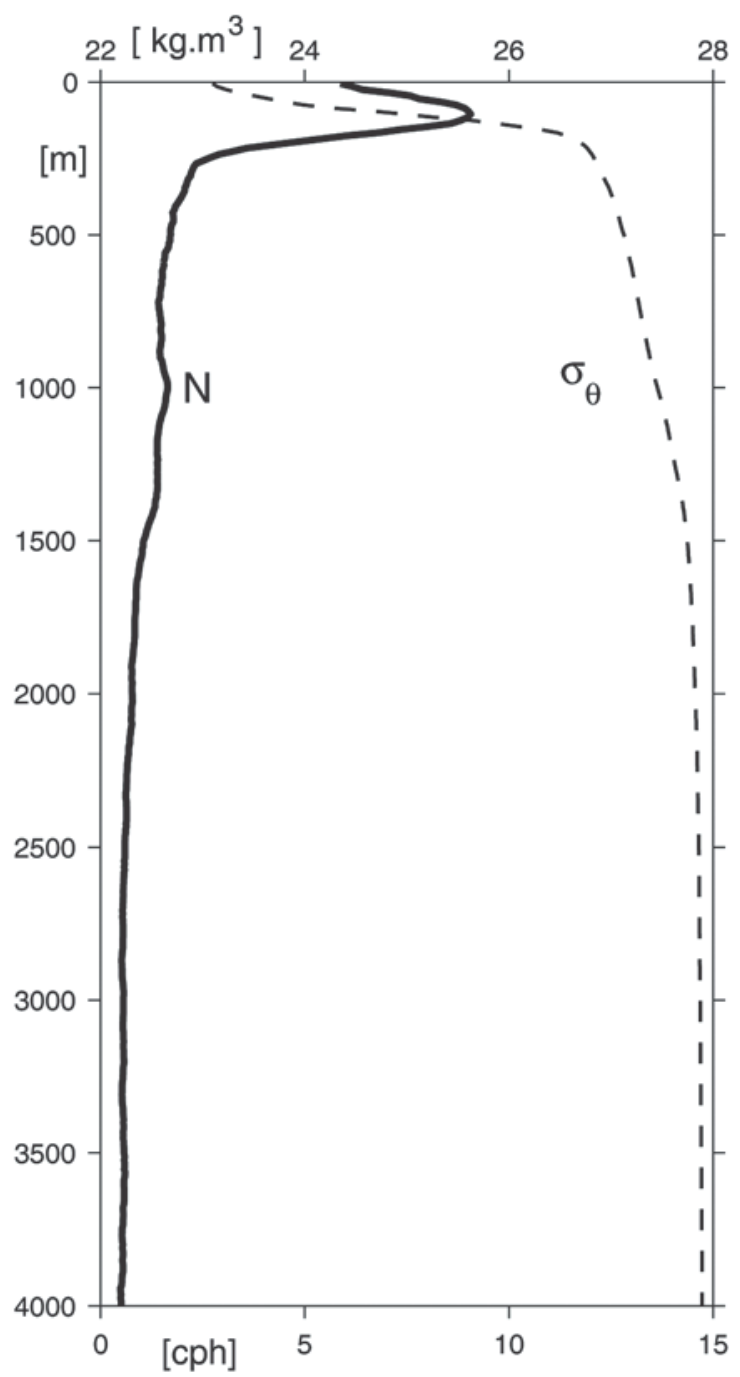

Figura 3 - Perfis Verticais da Freqüência de Brunt-Väisälä média anual (curva espessa) e Densidade Potencial (curva tracejada), representativos da seção de $44^{\circ} \mathrm{W}$.

Figure 3 - Vertical profiles of the four-cruise average Brunt-Väisälä frequency at $44^{\circ} \mathrm{W}$ (solid curve) and pontential density (dashed curve).

Em todos os cruzeiros, um pico acentuado confinado na parte mais superior da coluna de água e centrado em $125 \mathrm{~m}$, indica uma alta estratificação em subsuperfície. A presença da picnoclina permanente, típica de baixas latitudes (EMERY et al., 1983; HOURY et al., 1987), está representada pelo forte gradiente entre 2 e 9 ciclos por hora (cph).

0 perfil médio anual (Figura 4 - curva delgada) foi sobreposto aos perfis médios de cada cruzeiro como referência climatológica. Existem 
variações sazonais nos diferentes cruzeiros. 0 cruzeiro de set 90 apresenta o pico próximo de 10 cph, mantendo altos valores de estratificação até a superfície. Situação similar é encontrada no perfil de Jun91, porém, próximo à superfície, a frequiência cai rapidamente de 10 para 6 cph.
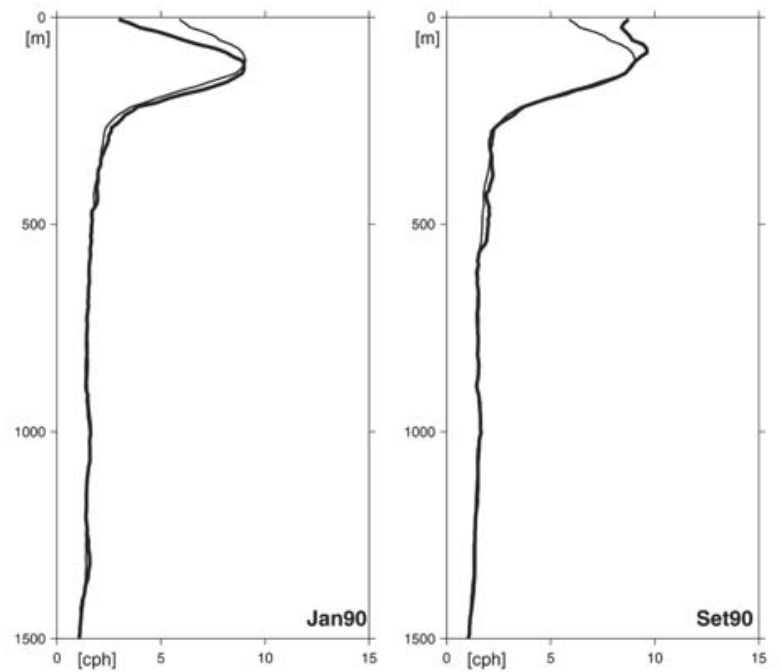

Em todos os cruzeiros, em regiões mais profundas que 250 metros, encontram-se valores de frequêencia tão baixos quanto 2 cph, permanecendo até o fundo. As curvas sazonais se sobrepõem à curva média anual nestas profundidades.

Figura 4 - Perfis Verticais da Freqüência de Brunt-Väisälä representativos da seção de 44 W para cada cruzeiro (curva espessa) sobrepostos no perfil anual (curva delgada).

Figure 4 - Vertical profiles of the Brunt-Väisälä frequency at 44W for each WESTRAX cruise individually (thick curves).

The annual curve (thin curve) is superimposed for comparison.

Segundo Emery e outros (1983) e Houry e outros (1987), regiões de médias e altas latitudes apresentam variabilidade sazonal de grande amplitude em contraposição às regiões de baixas latitudes. Desse modo, seria adequado, então, assumir uma estratificcação básica para o conjunto de dados WESTRAX? Para verificar se as oscilações sazonais descritas anteriormente, assim como a proximidade do equador, afetam as amplitudes modais e os raios de deformação, impedindo a aplicação do perfil médio, apresentamos a próxima seção.

\section{RAIOS DE DEFORMAÇÃO E MODOS VERTICAIS}

Os autovalores $\lambda_{\mathrm{i}}$ os quais formam um conjunto infinito porém discreto, são relacionados aos raios de deformação internos $\mathrm{Rd}_{\mathrm{i}}$ por:

$$
\operatorname{Rd}_{\mathrm{i}}=\left(\mathrm{f}_{0}^{2} \lambda_{\mathrm{i}}\right)^{-1 / 2}
$$

Desta forma, para cada raio de deformação interno existe um autovalor e um automodo vertical associados. A Tabela 2 apresenta os valores dos raios de deformação internos para os primeiros sete modos baroclínicos obtidos para cada cruzeiro e também para o perfil médio anual. Os valores de $\mathrm{Rd}_{i}$ dos dois primeiros modos baroclínicos variam cerca de 3,5\% da média ao longo do ano, enquanto os raios dos modos de maior ordem $(3-7)$ praticamente inexistem. Os valores de raios obtidos a partir do perfil médio anual refletem esta similaridade, e sugerem pouca sensitividade ao cálculo relativamente às variações de $\mathrm{N}$ na porção superior da coluna de água.

Como a região de estudo apresenta-se dentro do cinturão equatorial $\left( \pm 1,5^{\circ}\right.$ de latitude), a seção hidrográfica foi separada em duas partes, afim de calcular os raios de deformação internos e automodos normais para duas faixas de latitudes separadas pelo valor de $5^{\circ} \mathrm{N}$. A parte mais próxima do equador utilizou o valor de $\theta_{0}$ centrado em $3^{\circ} \mathrm{N}$ enquanto a segunda faixa utilizou um $\theta_{0}$ em $7^{\circ} \mathrm{N}$. A Tabela 3 mostra a variação dos valores de $\mathrm{Rd}_{\mathrm{i}}$ nas diferentes faixas de latitude em comparação com os valores obtidos para a seção completa, compreendida entre $0^{\circ}$ e $10^{\circ} \mathrm{N}$. Para este cálculo, foi utilizado o perfil médio anual da freqüência de estratificação. A escala dos movimentos, para o primeiro modo baroclínico, varia de $300 \mathrm{~km}$ na região mais ao sul da seção $\left(\theta_{0}\right.$ de $3^{\circ} \mathrm{N}$ ), vale aproximadamente $200 \mathrm{~km}$ para um $\theta_{0}$ de $5^{\circ} \mathrm{N}$, e cai para 
Tabela 2 - Os sete primeiros raios de deformação baroclínicos, expressos em $\mathrm{km}$, relativamente à latitude de $5^{\circ} \mathrm{N}$. Table 2 - The seven first baroclinic deformation radii (in $\mathrm{km}$ ) relative to a central latitude of $5^{\circ} \mathrm{N}$.

\begin{tabular}{cccccc}
\hline Modos BC & Jan90 & Set90 & Jan91 & Jun91 & Anual \\
\hline 1 & 202 & 201 & 197 & 195 & 197 \\
2 & 128 & 127 & 125 & 119 & 123 \\
3 & 78 & 78 & 77 & 78 & 75 \\
4 & 58 & 59 & 55 & 58 & 56 \\
5 & 46 & 47 & 45 & 45 & 45 \\
6 & 40 & 40 & 39 & 39 & 39 \\
7 & 34 & 34 & 33 & 34 & 33 \\
\hline
\end{tabular}

um valor da ordem de $100 \mathrm{~km}$ para a região mais ao norte da seção $\left(\theta_{0}\right.$ de $\left.7^{\circ} \mathrm{N}\right)$. Isto é decorrente da variação de $\mathrm{f}_{0^{\prime}}$ inversamente proporcional à $\operatorname{Rd}_{\mathrm{i}}$ na Equação (8).

Assim como para cada raio de deformação existe um autovalor associado, também existe um autovetor ou modo $\left(\mathrm{F}_{\mathrm{i}}(\mathrm{z})\right)$ correspondente. A Figura 5 apresenta um painel composto pelo perfil da freqüência de estratificação em toda coluna de água em conjunto com os sete primeiros automodos baroclínicos (linha cheia). As linhas tracejadas e pontilhadas são os modos calculados para as faixas de latitude mais próximas e mais distantes do equador, respectivamente. Fica evidente que a estrutura modal não se altera devido a proximidade do equador, apenas os raios baroclínicos crescem em função do decréscimo do parâmetro de Coriolis.

Tabela 3 - Os sete primeiros raios de deformação baroclínicos, expressos em $\mathrm{km}$, por faixas de latitude: 0 - $10^{\circ} \mathrm{N}$ (latitude central em $5 \mathrm{~N}$ ), 0 - $5^{\circ} \mathrm{N}$ (latitude central em 3N), 5 - 10 $\mathrm{N}$ (latitude central em 7N).

Table 3 - The seven first barodinic deformation radii (in $\mathrm{km}$ ) calculated by latitude ranges: $0-10^{\circ} \mathrm{N}$ (central latitude: $5^{\circ}$ ), 0-5 $\mathrm{N}$ (central latitude: $3^{\circ} \mathrm{N}$ ), and 5-10\% (central latitude: $\left.7^{\circ} \mathrm{N}\right)$.

\begin{tabular}{cccc}
\hline Modos BC & $\begin{array}{c}0^{\circ}-10^{\circ} \mathbf{N} \\
\left(\theta_{0}=5^{\circ} \mathrm{N}\right)\end{array}$ & $\begin{array}{c}0^{\circ}-5^{\circ} \mathbf{N} \\
\left(\theta_{0}=3^{\circ} \mathrm{N}\right)\end{array}$ & $\begin{array}{c}5^{\circ}=10^{\circ} \mathbf{N} \\
\left(\theta_{0}=7^{\circ} \mathrm{N}\right)\end{array}$ \\
\hline 1 & 197 & 334 & 138 \\
2 & 123 & 209 & 87 \\
3 & 75 & 127 & 54 \\
4 & 56 & 95 & 40 \\
5 & 45 & 75 & 33 \\
6 & 39 & 65 & 28 \\
7 & 33 & 55 & 23 \\
\hline
\end{tabular}

\section{RECOMPOSIÇÃO MODAL DOS PERFIS DE VELOCIDADE}

A recomposição dos perfis de velocidade, usando um número finito de modos, foi realizada através da equação (LEBLOND; MYSAK, 1978):

$\mathrm{u}^{*}=\sum_{\mathrm{i}=0}^{\mathrm{n}}\left(\mathrm{U}_{\mathrm{i}}\right) \mathrm{F}_{\mathrm{i}}, \quad \mathrm{n}=1,2, \ldots, 7$ onde $\mathrm{u}^{*}$ é a componente zonal de velocidade recomposta; $\mathrm{n}$ corresponde ao modo dinâmico em que a somatória é truncada. Seções de velocidade ao longo de $44^{\circ} \mathrm{W}$ foram construídas através destes perfis recompostos utilizando até oito modos dinâmicos, ou seja, o modo barotrópico $(n=0)$ e os sete primeiros modos baroclínicos $(n=1,2, \ldots, 7)$.

Para identificar os modos dinâmicos que individualmente respondem pela estrutura de velocidade, bem como em termos da média ponderada destes modos na recomposição da estrutura do escoamento, 

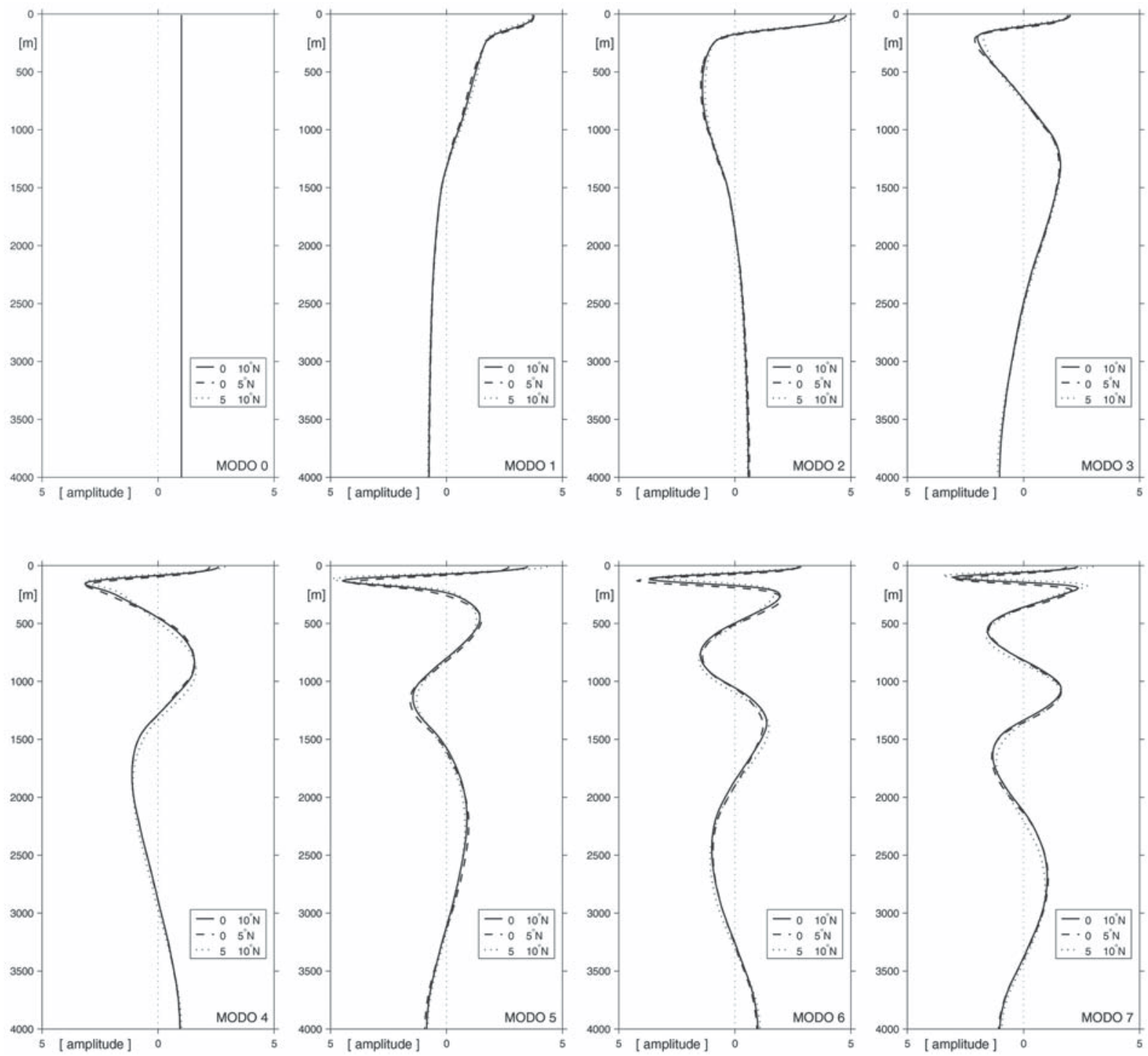

Figura 5 - Estruturas verticais dos modo barotrópico e primeiros sete modos baroclínicos calculados para as faixas de latitude de 0 - $10^{\circ} \mathrm{N}$ (linha cheia), 0 - 5N (linha tracejada) e 5 - 10 $\mathrm{N}$ (linha pontilhada).

Figure 5 - Vertical structure of the barotropic and first seven baroclinic modes by latitude ranges: 0-10 $\mathrm{N}$ (solid curves), 0-5 N (dashed curves), and 5-10 (dotted curves).

foram calculadas as raízes do erro médio quadrático (remq) seguindo formalismo apresentado por Pinardi e Robinson (1987),

remq $=\sqrt{\frac{\left\langle\left(u-u^{*}\right)^{2}\right\rangle}{\left\langle u^{2}\right\rangle}}$,

No entanto, valores de remq expressos em percentuais são mais intuitivos e foram calculados utilizando a relação: remq $\%=(1-$ remq $) \times 100$.

A Tabela 4 apresenta os valores das raízes do erro-médio quadrático calculados para os perfis truncados com os oito modos dinâmicos.

Painéis com as seções da componente zonal de velocidade reconstruída para cada cruzeiro são apresentados nas figuras 6 a 9. Os 
Tabela 4 - Raízes do erro-médio quadrático (remq), e seus respectivos valores percentuais, para cada modo dinâmico individualmente e para a soma acumulativa até o sétimo modo baroclínico.

Table 4 - Root mean square error (remq) and corresponding percentual values for each dynamical mode individually and for the cumulative sum of the first eight modes.

\begin{tabular}{c|ccccccccc}
\hline \multirow{2}{*}{ Modos Dinâmicos } & \multicolumn{3}{|c}{ Jan90 } & \multicolumn{2}{c}{ Set90 } & \multicolumn{2}{c}{ Jan91 } & \multicolumn{2}{c}{ Jun91 } \\
\cline { 2 - 9 } & remq & $\%$ & remq & $\%$ & remq & $\%$ & remq & $\%$ \\
\hline BT & 0.86 & 13.1 & 0.94 & 5.7 & 0.93 & 6.7 & 0.96 & 3.1 \\
1BC & 0.64 & 35.8 & 0.53 & 46.2 & 0.94 & 5.0 & 0.65 & 34.1 \\
2BC & 0.97 & 2.6 & 0.87 & 12.9 & 0.77 & 22.4 & 0.78 & 21.3 \\
3BC & 0.96 & 3.3 & 0.98 & 1.3 & 0.94 & 5.5 & 0.98 & 1.1 \\
4BC & 0.93 & 6.2 & 0.96 & 3.6 & 0.94 & 5.7 & 0.95 & 4.3 \\
5BC & 0.98 & 1.3 & 0.98 & 1.8 & 0.92 & 7.8 & 0.99 & 0.5 \\
6BC & 0.98 & 1.6 & 0.99 & 0.2 & 0.99 & 0.1 & 0.99 & 0.1 \\
7BC & 0.99 & 0.3 & 0.99 & 0.1 & 0.98 & 1.4 & 0.99 & 0.6 \\
\hline 2MD & 0.62 & 37.9 & 0.56 & 43.8 & 0.90 & 9.6 & 0.69 & 30.2 \\
3MD & 0.58 & 41.5 & 0.36 & 63.6 & 0.68 & 31.2 & 0.37 & 62.7 \\
4MD & 0.53 & 46.1 & 0.33 & 66.9 & 0.62 & 37.4 & 0.35 & 64.9 \\
5MD & 0.38 & 61.0 & 0.24 & 75.5 & 0.54 & 45.8 & 0.23 & 76.6 \\
6MD & 0.35 & 64.8 & 0.17 & 82.4 & 0.37 & 62.8 & 0.21 & 78.3 \\
7MD & 0.27 & 72.3 & 0.16 & 83.7 & 0.35 & 64.7 & 0.21 & 78.7 \\
8MD & 0.26 & 73.1 & 0.15 & 84.5 & 0.32 & 67.7 & 0.17 & 82.4 \\
\hline
\end{tabular}

campos exibem a recomposição modal (Equação (9)) de dois até oito modos dinâmicos (modo barotrópico e sete primeiros modos baroclínicos) em comparação com a seção de velocidade observada por PEGASUS (painel mais inferior à direita). As isotacas apresentam intervalo de $0.1 \mathrm{~m} \mathrm{~s}^{-1}$. Valores positivos indicam fluxo para leste e negativos para oeste. A posição das estações é indicada pelos círculos desenhados na parte superior dos campos.

Para 0 cruzeiro de jan90, o campo composto por dois modos dinâmicos (Figura 6 - 2MODOS) apresenta feições pobres e com valores de velocidade baixos. 0 modo barotrópico (BT) responde individualmente por 13,1\% do sinal medido por PEGASUS e o primeiro modo baroclínico (1BC) por 35,8\% (Tabela 4). Os dois primeiros modos dinâmicos (2MD) respondem juntos por apenas 37,9\%. Tanto o campo truncado em três modos (Figura 6 - 3MODOS), quanto aquele em quatro modos (Figura 6 - 4MODOS), ainda não conseguem reproduzir o núcleo de subsuperfície da SNE. Isto está relacionado à pequena importância dinâmica do segundo e do terceiro modos baroclínicos, que respondem, respectivamente por $2,6 \%$ e 3,3\%. Forte melhora da reprodução do sinal acontece quando a somatória modal inclui o quarto modo baroclínico $(4 B C)$, 0 qual explica 6,2\% do sinal medido. 0 campo de cinco modos (Figura 6 - 5MODOS), explica 61,0\% da variabilidade da estrutura vertical. Essa diferença é evidenciada tanto pela comparação qualitativa entre 0 campos truncados em quatro e em cinco modos (Figura 6), quanto quantitativamente pelo salto de 46,1\% para 61,0\% na Tabela 4. Os modos baroclínicos de maior ordem contribuem com valores inexpressivos inferiores a $2 \%, 0$ que pode ser constatado pelo fato de não haver grandes diferenças entre os campos truncados em 5, 6, 7 e 8 modos.

Para 0 cruzeiro de set90 (Figura 7), a contribuição dos modos manteve o mesmo comportamento. 0 1BC domina com 46,2\% seguido pelo 2BC com 12,9\%. 0 4BC é mais alto que $03 B$. Porém, o modo BT mostra o menor valor entre todos os cruzeiros analisados. Os três primeiros modos dinâmicos (3MD), respondem, então, por $66,9 \%$ da estrutura vertical. Com a significante colaboração do $4 B C$, salta para 0 expressivo valor de $75,5 \%$. Chega a $84,5 \%$ do sinal medido utilizando os oito primeiros modos dinâmicos, que é o mais alto valor percentual da tabela. Mais uma vez, o núcleo de subsuperfície do sistema CCNE-SNE somente é recomposto após a inclusão do quarto modo baroclínico.

Entretanto, para 0 cruzeiro de jan91 (Figura 8), a estrutura dinâmica mostra-se mais complexa e diferente daquela do cruzeiro jan90. Este fato foi inesperado, uma vez que ambos ocorreram exatamente na mesma estação: 0 inverno boreal. Nesse cruzeiro, é o segundo modo baroclínico aquele com maior amplitude, respondendo individualmente 
por $22,4 \%$. 0 BT e IBC responderam por inexpressivos 6,7\% e 5,0\%, respectivamente. 0 5BC é responsável por 7,8\% do sinal perfilado. 0 núcleo da SNE só atinge valores superiores a $0,5 \mathrm{~m} \mathrm{~s}$ no campo recomposto por seis modos dinâmicos. Todos os outros modos entre 0 BT e $05 \mathrm{BC}$ respondem com o mesmo grau de importância, dando a sensaçãa de que a energia do sistema foi espalhada nos primeiros modos dinâmicos, deixando o segundo modo dominar. 0 campo composto por oito modos dinâmicos explicou $67,7 \%$ da variabilidade do sinal amostrado. Foi o menor valor percentual entre os campos recompostos por oito modos dinâmicos. Isto indica que mais energia está espalhada em modos de maior ordem que não foram levados em consideração neste estudo.

Novamente, para 0 cruzeiro de jun9l (Figura 9), o IBC é dominante, respondendo por $34,1 \%$. De modo similar ao cruzeiro de set90, o segundo e o quarto modos baroclínicos se mostram importantes, respondendo por 21,3\% e 4,3\%, respectivamente. 0 modo BT aparece com seu menor valor entre todos os cruzeiros, 3,1\%. Todos os outros modos colaboram com valores muito baixos, incapazes de ultrapassar valores de $1,1 \%$. Da análise qualitativa podemos perceber que o núcleo de subsuperfície da CCNE é dissolvido após a inclusão do 7BC.

\section{CONCLUSÕES}

Utilizando perfilagens simultâneas de velocidade, salinidade e temperatura, o Método de Análise dos Modos Normais foi aplicado na região da CCNE/SNE. Os perfis da componente zonal de velocidade foram recompostos e comparados com o sinal medido. Análises qualitativas e quantitativas com reconstituiçãa por truncamento a um número finito $\mathrm{e}$ crescente de modos dinâmicos, baseadas em figuras de mérito estatísticas, forneceram informações necessárias para responder as seguintes questões: a) São os primeiro e segundo modos baroclínicos, como dito por Philander (1989), mais relevantes que o modo barotrópico na região equatorial do Oceâno Atlântico Tropical Oeste? b) É a afirmação de Philander e Pacanowski (1980) de que o segundo modo baroclínico é dominante realmente válida? Pode este modo ser usado como representante da estrutura dinâmica para qualquer porção do Oceano Atlântico Tropical? c) Os três primeiros modos dinâmicos (barotrópico e dois primeiros baroclínicos) foram suficientes para reproduzir de forma satisfatória 0 processo de retroflexão do sistema CNB (SILVEIRA et al., 2000). 0 mesmo pode ser aplicado para o sistema de correntes zonais ao longo de $44^{\circ} \mathrm{W}$ ? d) De que forma o núcleo de subsuperfície da CCNE, relatado por Bourlès e outros (1999), está relacionado à estrutura modal da região?

Nossos resultados confirmam a afirmação de Philander (1989) de que os dois primeiros modos baroclínicos são dominantes em regiões equatoriais. Para o cruzeiro de jun91, o modo barotrópico explica apenas $3,1 \%$ da estrutura vertical de velocidade observada contra os $34,1 \%$ e $21,3 \%$ dos primeiro e segundo modos baroclínicos, respectivamente. Apenas para o cruzeiro de jan90, o modo barotrópico apresentou maior importância $(13,1 \%)$.

Os resultados obtidos por Philander e Pacanowski (1980), através de modelagem numérica, indicaram que o segundo modo baroclínico é dominante devido ao fato de possuir maior amplitude em comparação com o primeiro e 0 terceiro modos baroclínicos, e conseqüentemente, pode representar a dinâmica do oceano equatorial em qualquer região da bacia atlântica. Nossos resultados mostram claramente que, ao longo de $44^{\circ} \mathrm{W}$, 0 segundo modo baroclínico não é dominante para três dos quatro cruzeiros analisados, e que para o cruzeiro de jan90, este modo foi responsável por apenas $2 \%$ da estrutura vertical do sinal de velocidade medido. Apenas para 0 cruzeiro de jan91, 0 segundo modo dinâmico apresentou maior importância que os demais, explicando 22,4\% do sinal de velocidade em $44^{\circ} \mathrm{W}$. Comparado com o primeiro modo baroclínico, que respondeu por aproximadamente 35\% nos cruzeiros de jan90 e jun91, e por $46 \%$ em jan91, o segundo modo baroclínico não domina. Este resultado corrobora a clássica afirmação de Lighthill (1969) de que o primeiro modo baroclínico domina a estrutura nos primeiros 100 metros de coluna de água.

A análise de modos normais revelou a existência de uma variaçã̃o sazonal relacionada com a estrutura de velocidade. Os cruzeiros de set90 e jun91 (outono e verão boreais) apresentaram uma dominância clara dos dois primeiros modos barodínicos, dando suporte a estudos prévios baseados em modelagem e teoria. De fato, os três primeiros modos dinâmicos foram responsáveis por $63 \%$ da estrutura de velocidade observada. Estes resultados concordam com os que Silveira et al. (2000) encontraram quando analisaram 0 cruzeiro de outono boreal (set90) para a região de retroflexão da CNB. Por outro lado, os cruzeiros de inverno boreal (jan90 e jan91) apresentaram um padrão de velocidade mais complexo. Para estes dois cruzeiros, são necessários seis (primeiros) modos dinâmicos para reproduzir 63\% da estrutura de velocidade observada. Os três primeiros modos dinâmicos responderam por 30\%$40 \%$ do sinal observado. Isto se deve ao fato de que, durante esses períodos, o processo de retroflexão da CNB não ocorreu, deixando de alimentar 0 sistema zonal de correntes equatoriais. Os valores de velocidade zonal são fracos e as CCNE e SNE apresentam-se separadas. Obviamente, quanto mais complexo o padrão de circulação, maior é 0 número de modos dinâmicos que detêm a energia da estrutura vertical.

A questão que se impõe: se a estrutura modal observada aqui, com participação significativa de modos de ordem mais alta, é resultado do fato de o meridiano de $44^{\circ} \mathrm{W}$ estar próximo à camada limite oeste do 

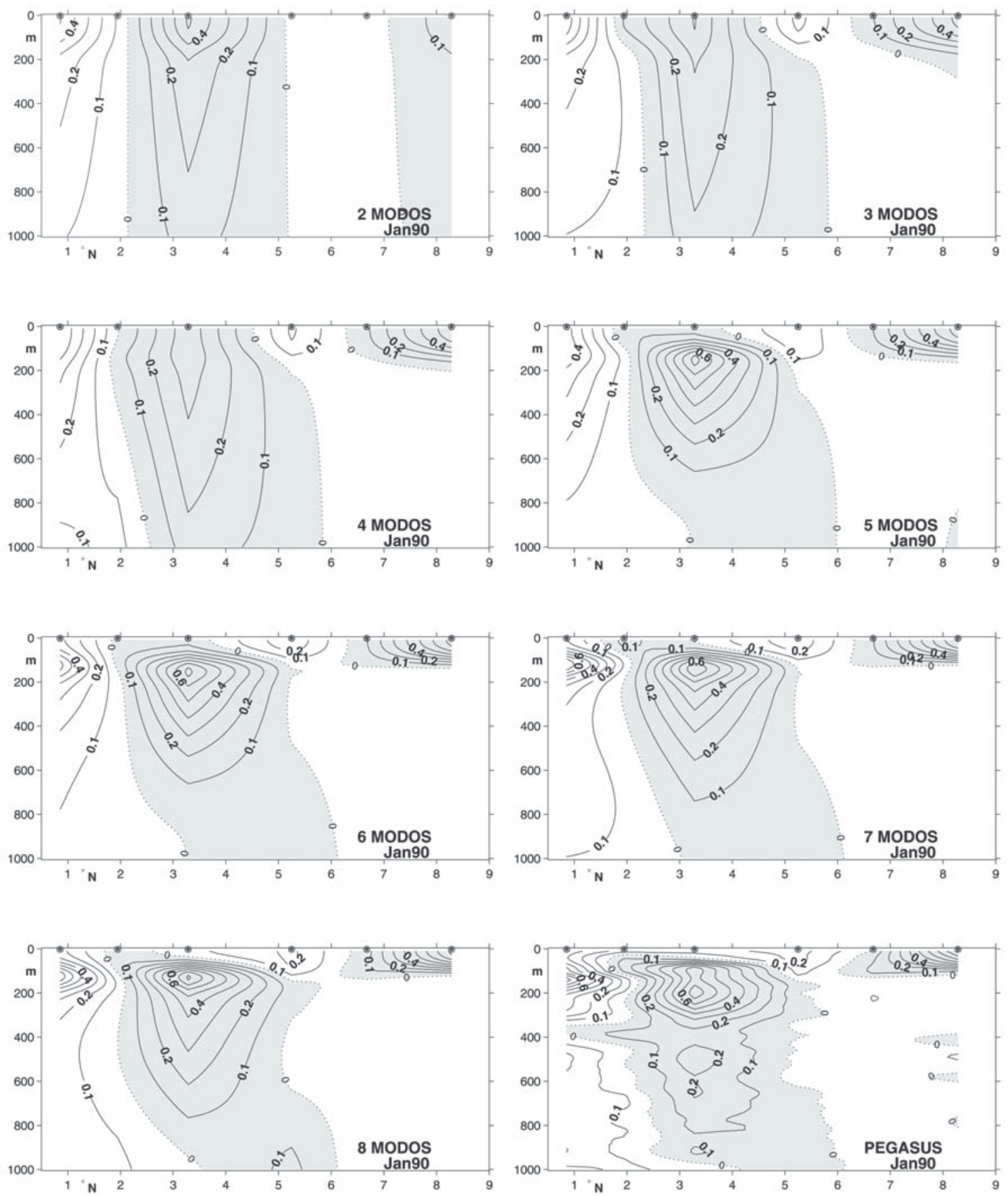

Figura 6 - Cruzeiro de jan90. Seções da componente zonal de velocidade (u) ao longo de 44 $4^{\circ} \mathrm{em} \mathrm{m} \mathrm{s}^{-1}$. As isotacas apresentam intervalo de $0.1 \mathrm{~m} \mathrm{~s}^{-1}$. Valores positivos indicam fluxo para leste e negativos para oeste. A profundidade está expressa em metros. A posição das estações é indicada pelos círculos desenhados na parte superior dos campos.

Figure 6 - Cruise jan90: sections of zonal velocity (u) at 44 W in $m s^{l}$. Positive values refer to eastward velocities, and negative values to westward velocities. The station locations are represented by black circles at $0 \mathrm{~m}$. 

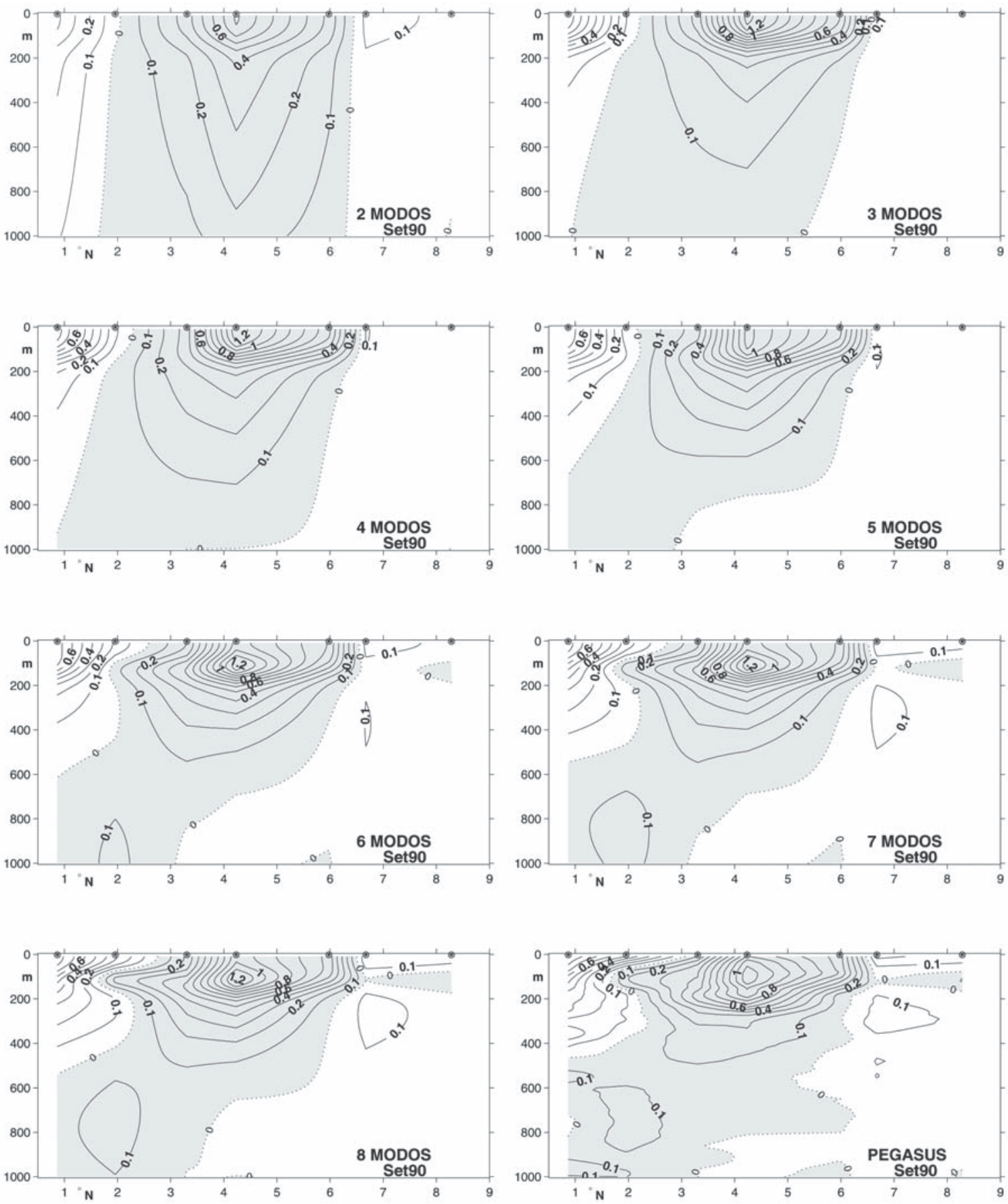

Figura 7 - Cruzeiro de set90. Seções da componente zonal de velocidade (u) ao longo de $44^{\circ} \mathrm{W}$ em m s.-1. As isotacas apresentam intervalo de $0.1 \mathrm{~m} \mathrm{~s}^{-1}$. Valores positivos indicam fluxo para leste e negativos para oeste. A profundidade está expressa em metros. A posição das estações é indicada pelos círculos desenhados na parte superior dos campos.

Figure 7 - Cruise sep90: sections of zonal velocity (u) at $44^{\circ} \mathrm{W}$ in $\mathrm{m} \mathrm{s}^{\mathrm{l}}$. Positive values refer to eastward velocities, and negative values to westward velocities. The station locations are represented by black circles at $0 \mathrm{~m}$. 

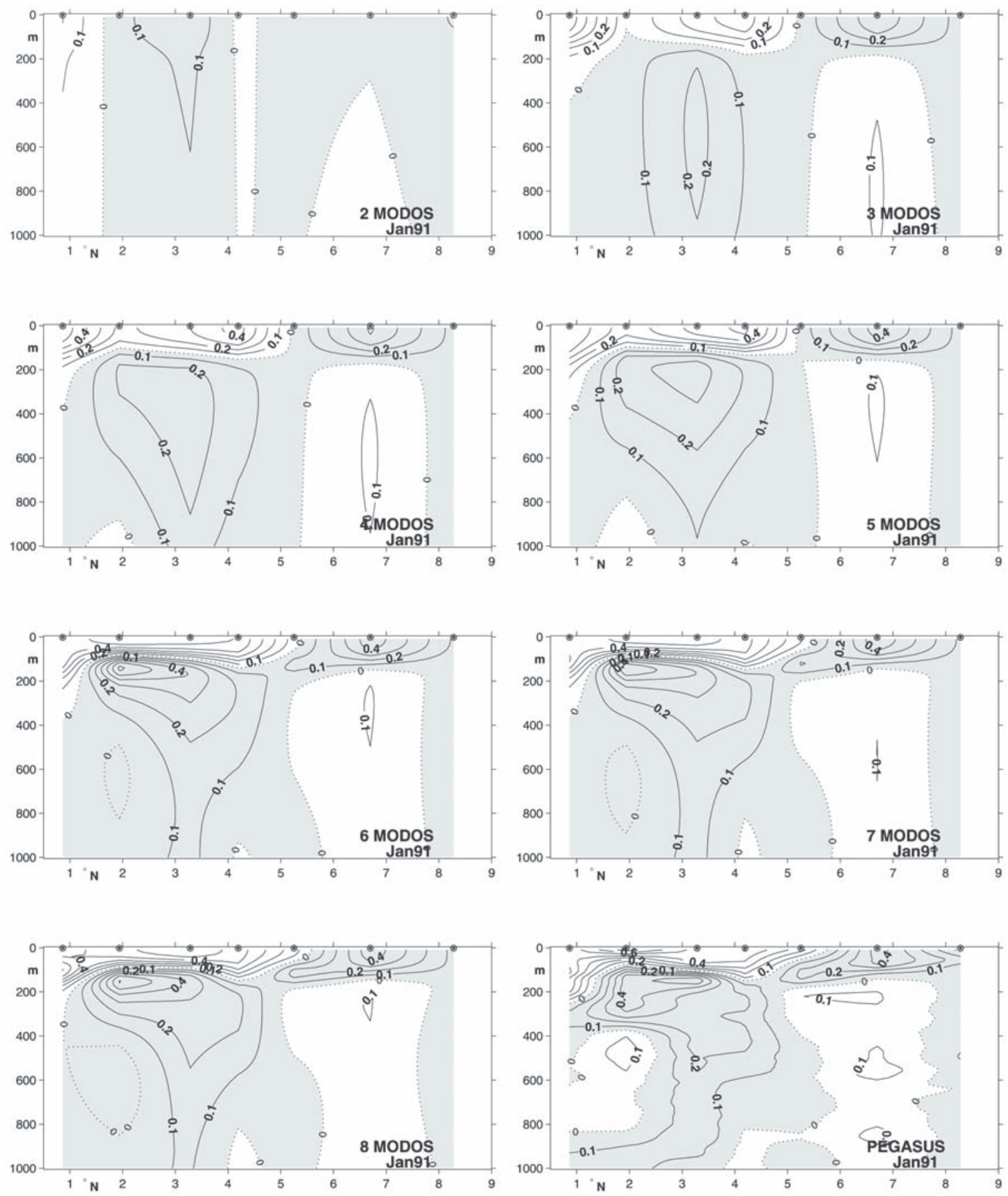

Figura 8 - Cruzeiro de jan91. Seções da componente zonal de velocidade (u) ao longo de 44 $4^{\circ} \mathrm{em} \mathrm{m} \mathrm{s}^{-1}$. As isotacas apresentam intervalo de $0.1 \mathrm{~m} \mathrm{~s}^{-1}$. Valores positivos indicam fluxo para leste e negativos para oeste. A profundidade está expressa em metros. A posição das estações é indicada pelos círculos desenhados na parte superior dos campos.

Figure 8 - Cruise jan91: sections of zonal velocity (u) at 44 W in $m s^{l}$. Positive values refer to eastward velocities, and negative values to westward velocities. The station locations are represented by black circles at $0 \mathrm{~m}$. 

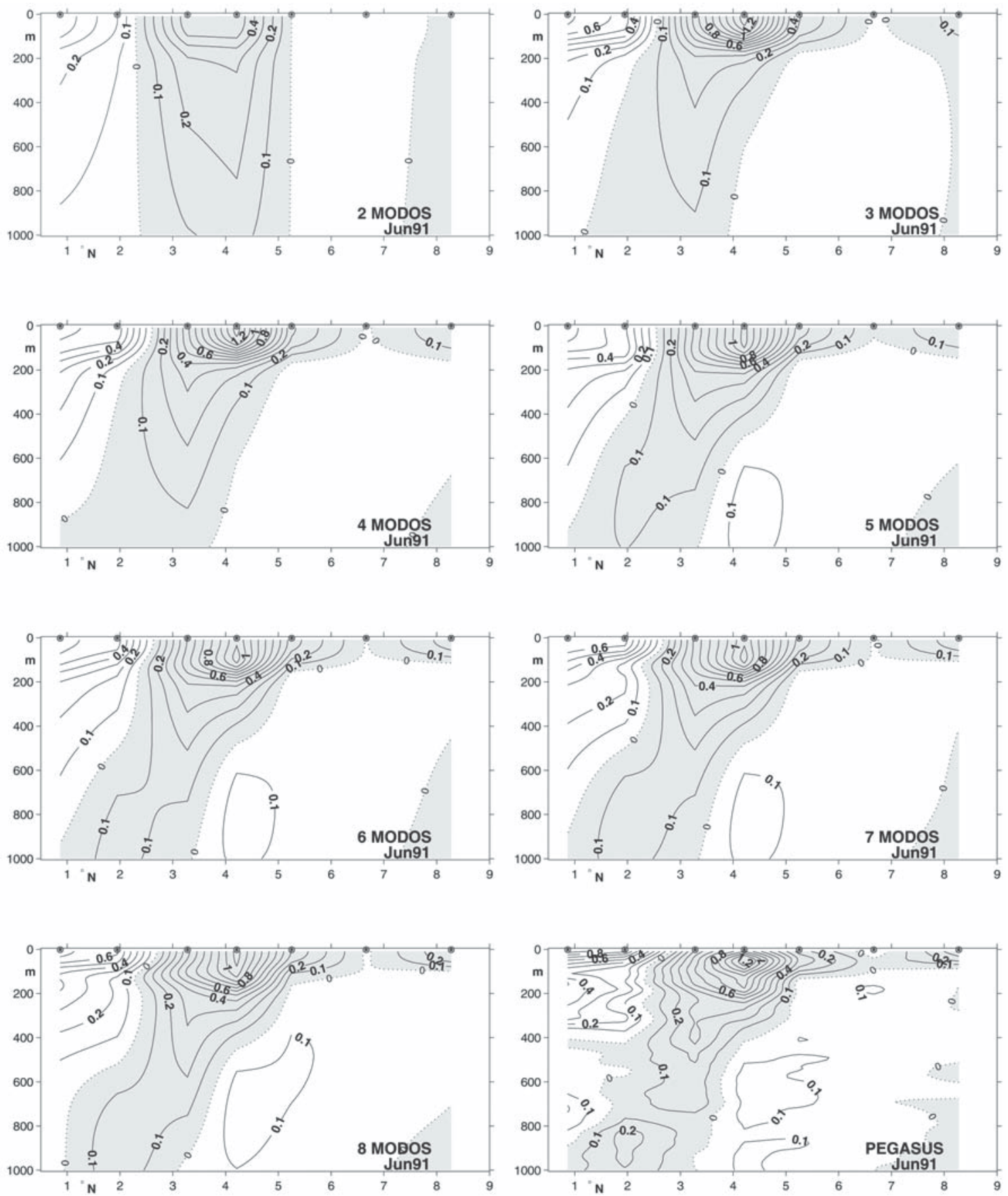

Figura 9 - Cruzeiro jun91. Seções da componente zonal de velocidade (u) ao longo de 44 $4^{\circ} \mathrm{em} \mathrm{m} \mathrm{s}^{-1}$. As isotacas apresentam intervalo de $0.1 \mathrm{~m} \mathrm{~s}^{-1}$. Valores positivos indicam fluxo para leste e negativos para oeste. A profundidade está expressa em metros. A posição das estações é indicada pelos círculos desenhados na parte superior dos campos.

Figure 9 - Cruise jun91: sections of zonal velocity (u) at 44W in $\mathrm{m} \mathrm{s}$. Positive values refer to eastward velocities, and negative values to westward velocities. The station locations are represented by black circles at $0 \mathrm{~m}$. 
Oceano Atlântico Tropical, e portanto, sítio de origem das CCNE e SNE. Comparação com a estrutura de velocidade observada em porções mais centrais da bacia oceânica, assim como a análise modal dessas, deve ser conduzida no intuito de confirmar os resultados aqui obtidos, que denotam maior complexidade dinâmica do que a teoria e a modelagem numérica aplicadas a região lhes conferiram até o momento.

\section{Agradecimentos}

Os autores expressam agradecimento ao Dr. Wendell Brown, por gentilmente ceder os dados WESTRAX, e à Fundação de Amparo à Pesquisa do Estado de São Paulo (FAPESP), pelos recursos concedidos através dos processos 2002/07292-2 e 2000/06985-9.

\section{REFERÊNCIAS}

Bourlès, B et al. Upper layer currents in the western tropical North Atlantic (1989-1991). Journal of Physical Oceanography, [S.I.], v. 104, n. Cl, 1361-1375, 1999.

BROWN, W.S. et al. A western tropical atlantic experiment (WESTRAX). Oceanography, [S.I.], v. 5, n. 1, p. 73-77, 1992.

CANE, M. The response of an equatorial ocean to simple wind-stress patterns, Parts I and II. Journal of Marine Research, [S.I.], n. 37, p. 233-299, 1979.

; SARACHIK, E. Forced baroclinic ocean motions, I. Journal of Marine Research, [S.I.], v. 34, n. 4, p. 629-665, 1976. . Forced baroclinic ocean motions, II. Journal of Marine Research, [S.I.], v. 35, n. 2, p. 395-432, 1977. . Forced baroclinic ocean motions, III. Journal of Marine Research, [S.I.], v. 37, p. 355-398, 1979.

EMERY, W. J.; LEE ,W. G.; MAGAARD, L. Geographic and seasonal distributions of Brunt-Väisälä frequency and Rossby Radii in the North Pacific and North Atlantic. Journal of Physical Oceanography, [S.I.], v. 14, p. 294-317, 1983.

HOURY, S. et al. Brunt-Väisälä Frequency and Rossby Radii in the South Atlantic. Journal of Physical Oceanography, [S.I.], v. 17, p. 1619-1626, 1987.
LEBLOND, P. H.; MYSAK, L. A. Waves in the ocean. [S.I.]: Elsevier, 1978. LIGHTHILL, M. J. Dynamics response of the Indian Ocean to the onset of the southwest monsoon. Philosophical Transactions of the Royal Society of London, [S.I.], v. 265, p. 45-92, 1969.

MCCLEAN, J. L.; KLINCK, J. M. Description and vorticity analysis of the 50-day oscillations in the western tropical Atlantic region of the CME model. Journal of Phisical Oceanography, [S.I.], v. 25, p. 2498-2517, 1995.

MCCREARY, J. P. A linear stratified ocean model of the Equatorial Undercurrent. Philosophical Transactions of the Royal Society of London, [S.I.], v. 298, p. 603-635, 1980. Series A, Mathematical and Physical Sciences.

PATTI, E. A geração dos anéis da Corrente Norte do Brasil devido ao forçamento pelo vento em um modelo isopicnal. 2001. $115 \mathrm{f}$. Tese (Doutorado)-Departamento de Oceanografia Física, Química e Geológica, Instituto Oceanográfico, Universidade de São Paulo, São Paulo, 2001. PHILANDER, S. G. H. El Niño La Niña, and the Southern oscillation. San Diego: Academic Press, 1989.

; PACANOWSKI, R. C. The Generation of Equatorial Currents.

Journal of Geophysical Research, [S.I.], v. 85, n. C2, p. 1123-1136, 1980.

PINARDI, N.; ROBINSON, A. R. Dynamics of deep thermocline jets in the POLYMODE region. Journal of Physical Oceanography, [S.I.], v. 17, p. 1163-1188, 1987.

SILVEIRA, I. C. A., BROWN, W. S.; FLIERL, G. R.. Dynamics of the North Brazil Current Retroflection from the WESTRAX observations. Journal of Geophysics Research, [S.I.], v. 105, n. C12, p. 28559-28583, 2000. SPAIN, P. F.; DORSON, D. L.; ROSSBY, H. T. PEGASUS: a simple, acoustically tracked, velocity profiler. Deep-Sea Research, [S.I.], v. 28A, n. 12, p. 1553-1567, 1981.

WILSON, W. D.; JOHNS, E.; MOLINARI R.L. Upper layer circulation in the western tropical North Atlantic Ocean during August 1989. Journal of Geophysics Research, [S.I.], v. 99, n. C11, p. 22, p. 22513-22523, 1994. 


\section{NOTAS SOBRE OS AUTORES}

Domingos Fernandes Urbano Neto é bacharel em Oceanologia na área de Gerenciamento Ambiental pela FURG, vinculado ao Laboratório de Oceanografia Física (de 1996 a 1999). Doutorando Direto no programa de Pós-Graduação em Oceanografía Física, desde 2000, pelo Instituto Oceanográfico da USP. Membro do Laboratório de Dinâmica Oceânica atua em estudos de circulação de meso-escala através de análise de dados e modelagem numérica. Bolsista-sanduíche CAPES, desde julho de 2003, no Department of Earth, Atmospheric, and Planetary Sciences do Massachusetts Institute of Technology (MIT), nos Estados Unidos, sob orientação da Profa. Dra. Paola MalanotteRizzoli e Dr. Markus Jochum.

Ilson Carlos Almeida da Silveira é professor-Doutor do Departamento de Oceanografia Física, Química e Geológica do Instituto Oceanográfico da Universidade de São Paulo, desde 1997. Graduado em Oceanografia pela UERJ, em 1984. Mestre em Oceanografia Física pelo IOUSP, em 1990. Nos EUA, Doutor pela University of New Hampshire, em 1996 e pós-doutoramento em Rhode Island, em 1997. Sua linha de pesquisa compreende a circulação de meso-escala do oceano, voltada para teoria de instabilidade hidrodinâmica e para 0 estudo da dinâmica das correntes de contorno oeste, principalmente da Corrente do Brasil, através do desenvolvimento e utilização de modelos analíicicos, semi-analíticos e numéricos. 
